\title{
Targeted Intraoperative Radiotherapy Tumour Bed Boost during Breast-Conserving Surgery after Neoadjuvant Chemotherapy
}

\author{
Jayant S. Vaidya Jeffrey S. Tobias \\ Division of Surgery and Interventional Science, University College London, London, United Kingdom
}

Kolberg and colleagues have recently published a series of papers [1-3] that, for the first time, give some idea of the outcomes of targeted intraoperative radiotherapy (TARGIT IORT) as a tumour bed boost during lumpectomy after neoadjuvant chemotherapy (NACT). The last of these series is published in the current issue of BREAST CARE. We would like to discuss these from two perspectives - NACT and TARGIT IORT.

\section{Neoadjuvant Chemotherapy}

The generally accepted aims of NACT are to downsize an inoperable cancer, and to assess tumour response, particularly within 'window-of-opportunity' translational research clinical trials.

Whether it is better to give systemic therapy before or after surgery has been assessed in 2 large meta-analyses of randomised trials of NACT versus adjuvant chemotherapy. In these metaanalyses, no difference in survival was found. However, they did find a higher risk of loco-regional recurrence in patients randomised to receive NACT before surgery when compared with adjuvant chemotherapy after surgery. The absolute increase in the risk of local recurrence was $3-5 \%$ at 5 years. The magnitude of the increased recurrence risk appears to be less with larger surgical excision after NACT and much higher when an operation is omitted altogether. The benefits of a smaller operation should, therefore, be balanced against the increased risk of local recurrence: at 5 years, about a $3 \%$ absolute increase of local recurrence vis-à-vis a $6 \%$ increased chance of avoiding a mastectomy $[4,5]$. The Early Breast Cancer Trialists group have performed a new meta-analysis with individual patient data from over 4,500 patients and these data have previously been presented but not published. They appear to reinforce the findings of the 2 preceding meta-analyses.

Therefore, if any of the benefits of NACT are to be realised, particularly the aim to convert a necessary mastectomy to breast con- servation, an additional treatment to improve local control would be welcome. If such an intervention has systemic benefits it would be an additional bonus.

\section{Systemic Effect of TARGIT IORT}

In the TARGIT-A trial [6-8], 3,451 patients eligible for breastconserving surgery for breast cancer were randomly allocated to receive either risk-adapted TARGIT IORT or whole breast external beam radiotherapy (EBRT). The TARGIT-IORT arm was found to be non-inferior to EBRT in terms of local control. In the analysis of deaths, there was no difference in breast cancer mortality, which was low in both arms of the trial. However, the trialists found a significantly lower non-breast cancer mortality for the TARGIT IORT patients (hazard ratio (HR) 0.47; $\mathrm{p}=0.0086$ ) (fig. 1). The irradiation of the lung, oesophagus and heart during tangential field radiation of the breast has been repeatedly shown to cause fatal cancers $[9,10]$ and deaths due to ischemic heart disease $[11,12]$. One would expect that TARGIT IORT should avoid such deaths. This view has been vindicated by a meta-analysis of randomised trials [13] of targeted radiotherapy/partial breast irradiation versus whole EBRT. This meta-analysis of over 4,500 patients found that in the breast cancer populations where the breast cancer mortality is low (about 5\%), the deleterious effect of whole breast radiation is unmasked and demonstrates an increased overall mortality. Thus, using targeted radiation rather than whole breast radiation in such patients leads to an improvement in overall survival albeit by a small absolute amount (fig. 2).

An interesting sub-group analysis performed in the TARGIT-A trial [14] was for survival from causes other than breast cancer, comparing TARGIT IORT followed by EBRT versus EBRT alone (fig. 3a). The group of patients who were randomised to TARGIT IORT but also received EBRT had a worse prognosis for breast cancer (as defined by the protocol) but would not be expected to be

\section{KARGER}

() 2017 S. Karger GmbH, Freiburg
Prof. Dr. Jayant S Vaidya, MD, FRCS, PhD 
Fig. 1. Kaplan-Meier plot for deaths from causes other than breast cancer (17 vs. 35 ) in the randomised TARGIT-A trial, showing a significantly higher risk of non-breast-cancer mortality with whole-breast fractionated radiation given postoperatively over several weeks (EBRT), compared with a risk-adapted single-dose radiation delivered to the tumour bed during the lumpectomy operation (TARGIT IORT) $[6,8]$.
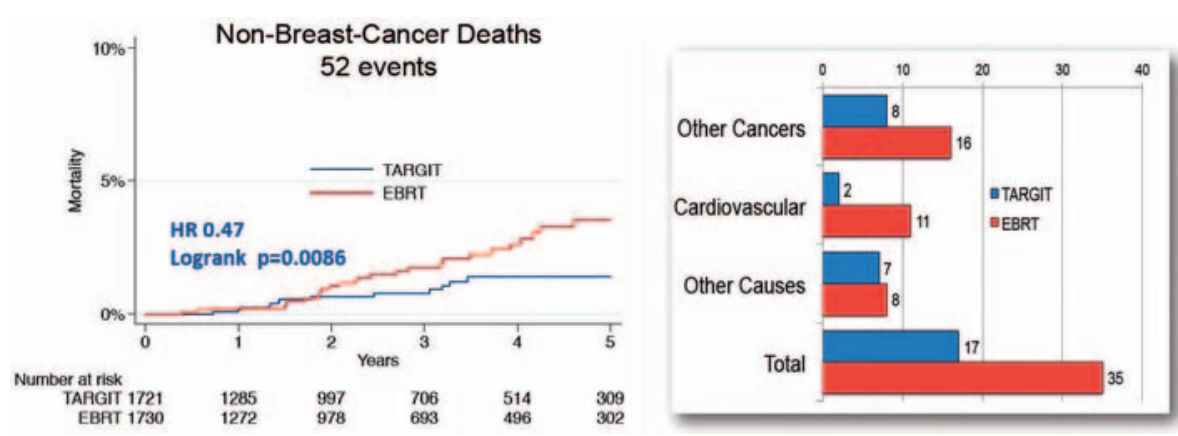

Fig. 2. Forest plots representing meta-analysis of difference in mortality between partial-breast irradiation (PBI) and whole-breast irradiation (WBI) with a random-effects model. The trials included for non-breast cancer (Non-BC) mortality were the Budapest trial, TARGIT-A, ELIOT, IMRT, and GEC-ESTRO. The median follow-up of all these trials was 5-6 years. Data from only the initial 1,222 patients in the TARGIT-A trial, whose median follow-up was 5 years, were included. The Budapest trial was not included in the analysis of $\mathrm{BC}$ deaths or total deaths because these figures were not available [14].

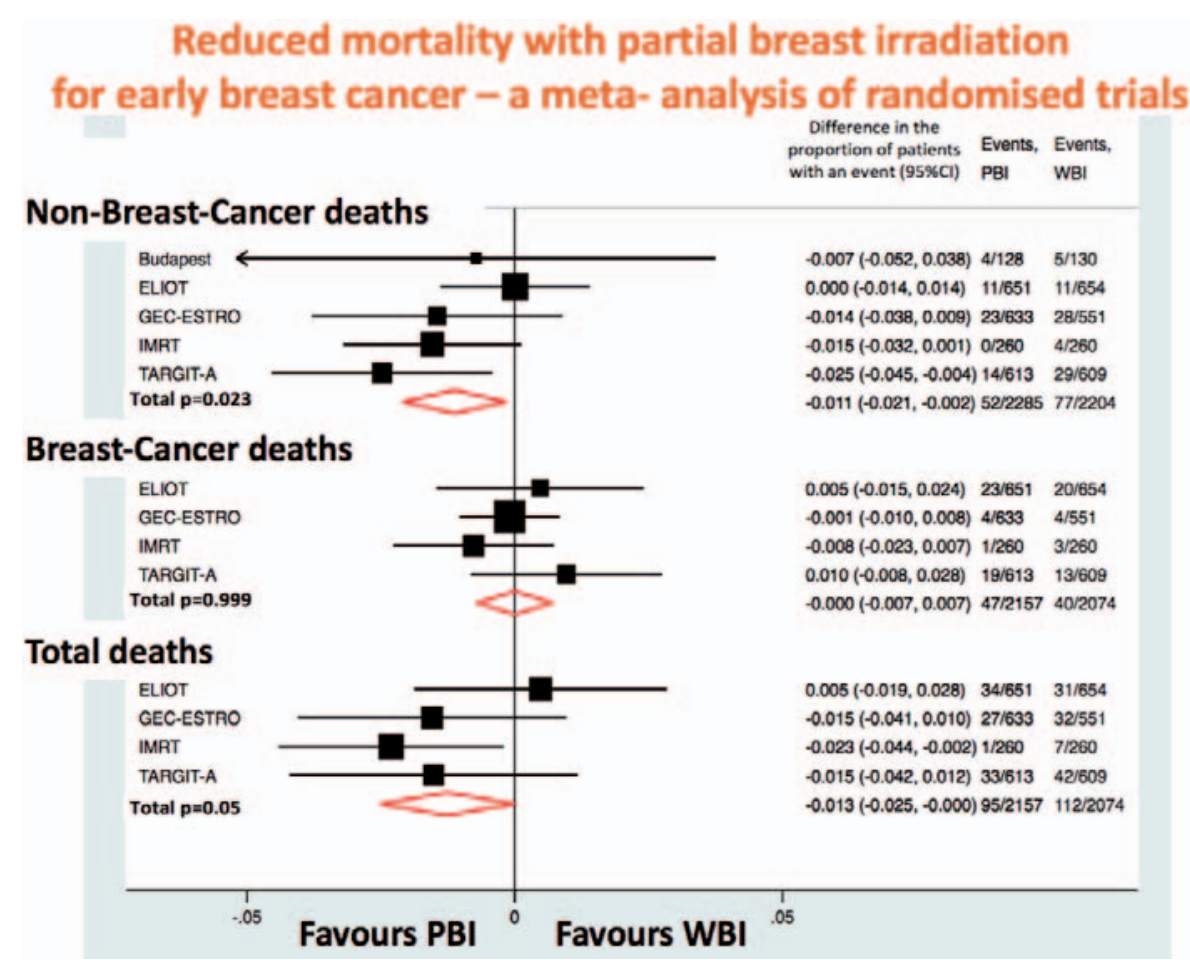

different from the rest of the cohort in terms of other co-morbidities; as both groups received EBRT, any difference found in non-breast-cancer mortality could be attributable to the delivery of TARGIT IORT during lumpectomy.

Remarkably, there were no deaths from non-breast cancer causes in the TARGIT + EBRT group compared with 24 in the EBRT group (0/218 vs. $24 / 892$, log-rank $\mathrm{p}=0.012)$. Although the numbers are small and the compared groups are not the randomised cohorts, these data suggest that avoidance of EBRT toxicity may not be the only reason for the fewer non-breast cancer deaths with TARGIT IORT, and the difference in mortality may also be attributable due to a beneficial systemic effect of TARGIT IORT. It leads to the hypothesis that the local effect of TARGIT on the tumour bed by inhibiting the cancer-stimulating cytokines, may spill over to reduce inflammatory response to trauma and have significant long-term systemic beneficial effects that might be protective against cardiac and cancer mortality [15].

Testing of this hypothesis within a randomised trial will occur in the TARGIT-B trial. In this trial, patients who are younger than
45 or at high risk of relapse, including those rendered suitable for breast conservation by pre-operative administration of NACT, are randomly allocated to receive a tumour bed boost either by TARGIT IORT during lumpectomy or by the traditional EBRT. While an improvement in local control is the primary aim, the specified second aim of this trial is to assess whether a TARGIT IORT boost has any effect on breast cancer and non-breast cancer survival.

\section{NACT + Surgery Including TARGIT IORT}

Kolberg and colleagues have reported the results of consecutive patients receiving TARGIT-IORT during lumpectomy after NACT [16]. As a comparator control, they used consecutive patients treated in the previous year without TARGIT IORT, and have taken efforts to ensure that the 2 groups are matched in all other aspects in this non-randomised study. To ensure that the follow-up periods were similar, they have truncated the follow-up for the historically older cohort. 
a

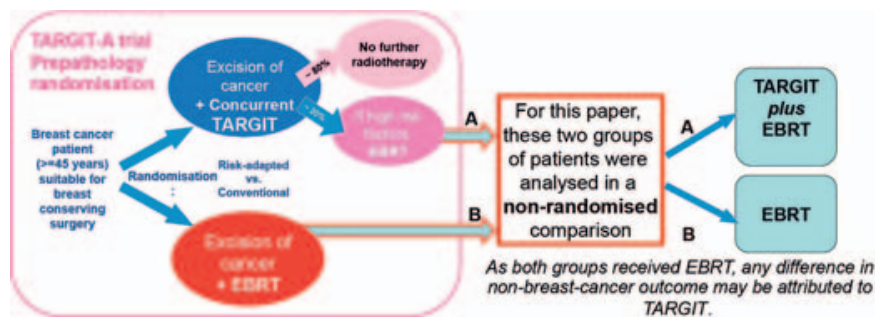

b

Deaths from causes other than breast cancer

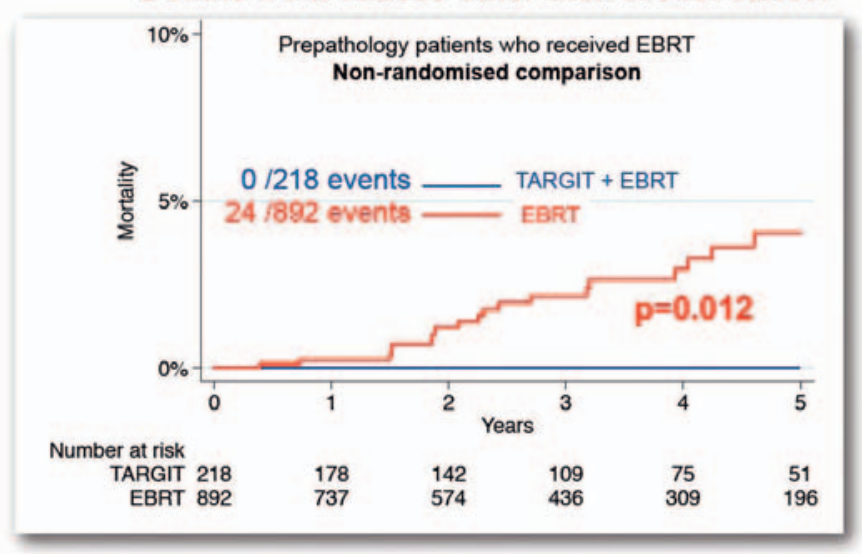

Fig. 3. Non-randomised comparison between those who received TARGIT IORT + EBRT and those who received EBRT for deaths from non-breast-cancer causes. a Schema of the analysed cohorts of patients; b Kaplan-Meier plot depicting deaths from causes other than breast cancer in prepathology patients who received EBRT; non-randomised comparison $[8,14,15]$.

In their first of the 3 papers they reported that patients receiving oncoplastic breast-conserving surgery after NACT along with TARGIT IORT boost, experienced similar local control, but statistically significantly better survival outcomes than those who re- ceived a postoperative EBRT boost (overall survival 96.7\% vs. $81.7 \%$, HR 0.19, log rank $\mathrm{p}=0.016$, and distant-disease-free survival $95.1 \%$ vs. 69.0, HR $0.23, \log$ rank $p=0.012$ ) [2]. In the subsequent papers, they tried to ascertain if there is any impact of tumour biology on this effect by analysing the data in 3 different subgroups: estrogen receptor-positive progesterone receptor-positive HER2-negative, HER2-positive, and triple-negative tumours. The results were numerically similar, but did not reach statistical significance, most likely due to paucity of events. It is arguable that such an analysis of the impact of tumour factors should normally be performed using a Cox proportional hazard model.

While these non-randomised data are not enough to prompt a change in practice, they are reassuring that, despite the short physical range of radiation field of TARGIT IORT, its effectiveness is at least as good as an externally delivered tumour bed boost and it may have a bonus of systemic beneficial effects. Therefore, at the very least these data give a strong support to clinicians and patients to participate in the TARGIT-B trial.

\section{Acknowledgement}

The authors thank Professor Michael Baum, Emeritus Professor of Surgery, University College London, for valuable discussions that contributed to the content of this manuscript.

\section{Disclosure Statement}

JSV has received a research grant from Photoelectron Corp (1996-99) and from Carl Zeiss for supporting data management at the University of Dundee (Dundee, UK) and has subsequently received honoraria.

For both authors, Carl Zeiss sponsors some of the travel and accommodation for meetings/conferences where targeted intraoperative radiotherapy is being presented.

\section{References}

1 Kolberg HC, Loevey G, Akpolat-Basci L, et al.: Targeted intraoperative radiotherapy tumour bed boost during breast conserving surgery after neoadjuvant chemotherapy in HER2 positive and triple negative breast cancer. Rev Recent Clin Trials 2017;12:93-100.

2 Kolberg HC, Loevey G, Akpolat-Basci L, et al.: Targeted intraoperative radiotherapy tumour bed boost during breast-conserving surgery after neoadjuvant chemotherapy. Strahlenther Onkol 2017;193:62-69.

3 Kolberg HC, Lövey G, Akpolat-Basci L, et al.: Targeted intraoperative radiotherapy tumour bed boost during breast-conserving surgery after neoadjuvant chemotherapy - a subgroup analysis of hormone receptorpositive HER2-negative breast cancer. Breast Care 2017;12:DOI: 10.1159/000479424.

4 Mauri D, Pavlidis N, Ioannidis JPA: Neoadjuvant versus adjuvant systemic treatment in breast cancer: a meta-analysis. J Natl Cancer Inst 2005;97:188-194.

5 Mieog JS, van der Hage JA, van de Velde CJ: Preoperative chemotherapy for women with operable breast cancer. Cochrane Database Syst Rev 2007:CD005002.
6 Vaidya JS, Wenz F, Bulsara M, et al.: Risk-adapted targeted intraoperative radiotherapy versus whole-breast radiotherapy for breast cancer: 5 -year results for local control and overall survival from the TARGIT-A randomised trial. Lancet 2014;383:603-613.

7 Vaidya JS, Bulsara M, Wenz F, et al.: Pride, prejudice, or science - attitudes towards the results of the TARGIT-A trial of targeted intraoperative radiotherapy for breast cancer. Int J Radiat Oncol Biol Phys 2015;92:491-497.

8 Vaidya JS, Wenz F, Bulsara M, et al.: An international randomised controlled trial to compare targeted intraoperative radiotherapy (TARGIT) with conventional post-operative radiotherapy after conservative breast surgery for women with early stage breast cancer (The TARGIT-A trial). Health Technol Assess 2016;20:1-188.

9 Grantzau T, Thomsen MS, Vaeth M, Overgaard J: Risk of second primary lung cancer in women after radiotherapy for breast cancer. Radiother Oncol 2014;111: 366-373.

10 Henson KE, McGale P, Taylor C, Darby SC: Radiationrelated mortality from heart disease and lung cancer more than 20 years after radiotherapy for breast cancer. Br J Cancer 2013;108:179-182.
11 Darby SC, Ewertz M, McGale P, et al.: Risk of ischemic heart disease in women after radiotherapy for breast cancer. N Engl J Med 2013;368:987-998.

12 Wang K, Eblan MJ, Deal AM, et al.: Cardiac toxicity after radiotherapy for stage III non-small-cell lung cancer: pooled analysis of dose-escalation trials delivering 70 to 90 Gy. J Clin Oncol 2017;35:1387-1394.

13 Vaidya JS, Bulsara M, Wenz F, et al.: Reduced mortality with partial-breast irradiation for early breast cancer: a meta-analysis of randomized trials. Int J Radiat Oncol Biol Phys 2016;96:259-265.

14 Vaidya JS, Bulsara M, Wenz F, et al.: The lower nonbreast cancer mortality with TARGIT in the TARGITA trial could be a systemic effect of TARGIT on tumor microenvironment. Int J Radiat Oncol Biol Phys 2013; 87:S240.

15 Vaidya JS, Bulsara M, Wenz F: Ischemic heart disease after breast cancer radiotherapy. N Engl J Med 2013; 368:2526-2527.

16 Kolberg H-C, Loevey G, Akpolat-Basci L, et al: Targeted intraoperative radiotherapy tumour bed boost during breast-conserving surgery after neoadjuvant chemotherapy. Strahlenther Onkol 2017;193:62-69. 